\title{
Hardy spaces for semigroups with Gaussian bounds
}

\author{
Jacek Dziubański ${ }^{1}$ • Marcin Preisner $^{1}$
}

Received: 3 July 2017 / Accepted: 16 October 2017 / Published online: 5 November 2017

(C) The Author(s) 2017. This article is an open access publication

\begin{abstract}
Let $T_{t}=e^{-t L}$ be a semigroup of self-adjoint linear operators acting on $L^{2}(X, \mu)$, where $(X, d, \mu)$ is a space of homogeneous type. We assume that $T_{t}$ has an integral kernel $T_{t}(x, y)$ which satisfies the upper and lower Gaussian bounds:

$$
\frac{C_{1}}{\mu(B(x, \sqrt{t}))} \exp \left(-c_{1} d(x, y)^{2} / t\right) \leq T_{t}(x, y) \leq \frac{C_{2}}{\mu(B(x, \sqrt{t}))} \exp \left(-c_{2} d(x, y)^{2} / t\right) .
$$

By definition, $f$ belongs to $H^{1}(L)$ if $\|f\|_{H^{1}(L)}=\left\|\sup _{t>0}\left|T_{t} f(x)\right|\right\|_{L^{1}(X, \mu)}<\infty$. We prove that there is a function $\omega(x), 0<c \leq \omega(x) \leq C$, such that $H^{1}(L)$ admits an atomic decomposition with atoms satisfying: supp $a \subset B,\|a\|_{L^{\infty}} \leq \mu(B)^{-1}$, and the weighted cancelation condition $\int a(x) \omega(x) \mathrm{d} \mu(x)=0$.
\end{abstract}

Keywords Hardy space - Maximal function - Atomic decomposition - Gaussian bounds . Hölder estimates

Mathematics Subject Classification Primary 42B30; Secondary 42B25 · 42B35 · 35J10

\section{Introduction}

Let $(X, d)$ be a metric space equipped with a nonnegative Borel measure $\mu$. We shall assume that $\mu(X)=\infty$ and $0<\mu(B(x, r))<\infty, r>0$, where $B(x, r)=\{y \in X \mid d(x, y) \leq r\}$ denotes the closed ball centered at $x$ and radius $r$. Suppose $(X, d, \mu)$ is a space of homoge-

The research was supported by the Polish National Science Center (Narodowe Centrum Nauki) Grant No. DEC-2012/05/B/ST1/00672.

$凶$ Marcin Preisner

marcin.preisner@uwr.edu.pl

Jacek Dziubański

jacek.dziubanski@math.uni.wroc.pl

1 Instytut Matematyczny, Uniwersytet Wrocławski, pl. Grunwaldzki 2/4, 50-384 Wrocław, Poland 
neous type in the sense of Coifman and Weiss [8], which means that the doubling condition holds, namely: There is $C>0$ such that $\mu(B(x, 2 r)) \leq C \mu(B(x, r))$ for $r>0$ and $x \in X$. It is well known that the doubling condition implies that there are $q>0$ and $C_{d}>0$ such that

$$
\mu(B(x, s r)) \leq C_{d} s^{q} \mu(B(x, r)) \text { for } x \in X, r>0 \text {, and } s \geq 1 .
$$

Suppose that $L$ is a nonnegative densely defined self-adjoint linear operator on $L^{2}(X, \mu)$. Let $T_{t}=e^{-t L}, t>0$, denote the semigroup of linear operators generated by $-L$. We impose that there exists $T_{t}(x, y)$, such that

$$
T_{t} f(x)=\int_{X} T_{t}(x, y) f(y) \mathrm{d} \mu(y) .
$$

Clearly, $T_{t}(x, y)=T_{t}(y, x)$ for $t>0$ and a.e. $x, y \in X$. Moreover, we assume the following lower and upper Gaussian bounds, that is, there are constants $c_{1} \geq c_{2}>0$ and $C_{0}>0$ such that

$$
\frac{C_{0}^{-1}}{\mu(B(x, \sqrt{t}))} \exp \left(-\frac{c_{1} d(x, y)^{2}}{t}\right) \leq T_{t}(x, y) \leq \frac{C_{0}}{\mu(B(x, \sqrt{t}))} \exp \left(-\frac{c_{2} d(x, y)^{2}}{t}\right)
$$

for $t>0$ and a.e. $x, y \in X$. It is well known that (1.3) implies that for every $n \in \mathbb{N}$ we have

$$
\left|\frac{\partial^{n}}{\partial t^{n}} T_{t}(x, y)\right| \leq \frac{C_{n} t^{-n}}{\mu(B(x, \sqrt{t}))} \exp \left(-\frac{c_{n}^{\prime} d(x, y)^{2}}{t}\right),
$$

for $t>0$ and a.e. $x, y \in X$ with some $C_{n}, c_{n}^{\prime}>0$. For this fact see, e.g., [20, (7.1)], [10,27].

The Hardy space $H^{1}(L)$ related to $L$ is defined by means of the maximal function of the semigroup $T_{t}$, namely

$$
H^{1}(L):=\left\{f \in L^{1}(X, \mu)\left|\|f\|_{H^{1}(L)}:=\left\|\sup _{t>0}\left|T_{t} f\right|\right\|_{L^{1}(X, \mu)}<\infty\right\} .\right.
$$

On the other hand, we define atomic Hardy spaces as follows. Suppose we have a space $X$ with a doubling measure $\sigma$ and a quasi-metric $\rho$. We call a function $a$ an $(\rho, \sigma)$-atom if there exists a ball $B=B_{\rho}\left(x_{0}, r\right):=\left\{x \in X \mid \rho\left(x, x_{0}\right) \leq r\right\}$ such that: supp $a \subseteq B$, $\|a\|_{\infty} \leq \sigma(B)^{-1}$, and

$$
\int_{B} a(x) \mathrm{d} \sigma(x)=0 .
$$

By definition, a function $f \in L^{1}(X, \sigma)$ belongs to $H_{\text {at }}^{1}(\rho, \sigma)$, if there exist $(\rho, \sigma)$-atoms $a_{k}$ and complex numbers $\lambda_{k}$, such that

$$
f=\sum_{k=1}^{\infty} \lambda_{k} a_{k} \quad \text { and } \quad \sum_{k=1}^{\infty}\left|\lambda_{k}\right|<\infty .
$$

If such sequences exist, we define the norm $\|f\|_{H_{\mathrm{at}}^{1}(\rho, \sigma)}$ to be the infimum of $\sum_{k=1}^{\infty}\left|\lambda_{k}\right|$ in the above presentations of $f$. Notice that in this paragraph we have changed the notation. This is because in the article we will use different metrics and measures.

For $x \in X$ let $\Phi_{x}:(0, \infty) \rightarrow(0, \infty)$ be the nondecreasing function defined by

$$
\Phi_{x}(t)=\mu(B(x, t)) .
$$

The first main result of this paper is the following. 
Theorem 1 Suppose that $(X, d, \mu)$ is a space of homogeneous type and assume that for each $x \in X$ the function $\Phi_{x}$ is a bijection on $(0, \infty)$. Let a nonnegative self-adjoint linear operator $L$ on $L^{2}(X, \mu)$ be given such that the semigroup $T_{t}=e^{-t L}$ satisfies (1.3). Then there exist a constant $C>0$ and a function $\omega$ on $X, 0<C^{-1} \leq \omega(x) \leq C$, such that the spaces $H^{1}(L)$ and $H_{\mathrm{at}}^{1}(d, \omega \mu)$ coincide and the corresponding norms are equivalent,

$$
C^{-1}\|f\|_{H_{\mathrm{at}}^{1}(d, \omega \mu)} \leq\|f\|_{H^{1}(L)} \leq C\|f\|_{H_{\mathrm{at}}^{1}(d, \omega \mu)} .
$$

Moreover, $\omega$ is L-harmonic, that is $T_{t} \omega=\omega$ for $t>0$.

Let us notice that the assumption on $\Phi_{x}$ implies that $\mu(X)=\infty$ and $\mu$ is nonatomic. This will be used later on. By definition, we call a semigroup conservative if

$$
\int_{X} T_{t}(x, y) \mathrm{d} \mu(y)=1
$$

for $t>0$ and $x \in X$.

Theorem 2 Suppose that $(X, d, \mu)$ is a space of homogeneous type and assume that for each $x \in X$ the function $\Phi_{x}$ is a bijection on $(0, \infty)$. Let a nonnegative self-adjoint linear operator $L$ on $L^{2}(X, \mu)$ be given such that the semigroup $T_{t}=e^{-t L}$ satisfies (1.3) and (1.6). Then, the spaces $H^{1}(L)$ and $H_{\mathrm{at}}^{1}(d, \mu)$ coincide and the corresponding norms are equivalent, i.e., there exists $C>0$ such that

$$
C^{-1}\|f\|_{H_{\mathrm{at}}^{1}(d, \mu)} \leq\|f\|_{H^{1}(L)} \leq C\|f\|_{H_{\mathrm{at}}^{1}(d, \mu)},
$$

It appears that Theorem 2 is equivalent to Theorem 1, see Sect. 3. Let us also emphasize that we do not require any regularity conditions on the kernels $T_{t}(x, y)$. However, it turns out that (1.3) implies Hölder-type estimates on $T_{t}(x, y)$, which are crucial for this paper. This will be discussed in Sect. 4 (see Theorem 5 and Corollary 14). In fact, Sect. 4 gives a short, independent, and self-contained proof of Hölder-type estimates of the heat kernel that satisfies (1.3), which can be interesting in its own right. Furthermore, although Theorems 1 and 2 are stated for the heat semigroup, we would like to emphasize that the same theorems can be proved for the Hardy space $H^{1}(\sqrt{L})$ associated with the Poisson semigroup $e^{-t \sqrt{L}}$ as well, see Theorem 4 and Remark 21 .

The theory of the classical Hardy spaces on the Euclidean spaces $\mathbb{R}^{n}$ has its origin in studying holomorphic functions of one variable in the upper half-plane. The reader is referred to the original works of Stein and Weiss [32], Burkholder et al. [6], Fefferman and Stein [15]. Very important contribution to the theory is the atomic decomposition of the elements of the $H^{p}$ spaces proved by Coifman [7] in the one-dimensional case and then by Latter [23] for $H^{p}\left(\mathbb{R}^{n}\right)$. The theory was then extended to the space of homogeneous type (see, e.g., [8,24, 33]). For more information concerning the classical Hardy spaces, their characterizations and historical comments we refer the reader to Stein [31]. A very general approach to the theory of Hardy spaces associated with semigroups of linear operators satisfying the Davies-Gaffney estimates was introduced by Hofmann et al. [20] (see also [1,30]). Let us point out that the classical Hardy spaces can be thought as those associated with the classical heat semigroup $e^{t \Delta}$. Finally we want to remark that the present paper takes motivation from $[13,14]$, where the authors studied $H^{1}$ spaces associated with Schrödinger operators $-\Delta+V$ on $\mathbb{R}^{n}, n \geq 3$, with Green bounded potentials $V \geq 0$.

In what follows $C$ and $c$ denote different constants that may depend on $C_{d}, q, C_{0}, c_{1}, c_{2}$. By $U \lesssim V$ we understand $U \leq C V$ and $U \simeq V$ means $C^{-1} V \leq U \leq C V$. 
The paper is organized as follows. In Sect. 2 we prove that estimate (1.3) implies the existence of an $L$-harmonic function $\omega$ such that $0<C^{-1} \leq \omega(x) \leq C$. The equivalence of Theorems 1 and 2 is a consequence of the Doob transform, see Sect. 3. Then a proof of Theorem 2 is given in a few steps. First, in Sect. 4 we prove Hölder-type estimates for a conservative semigroup. Then, in Sect. 5 we introduce a new quasi-metric $\widetilde{d}$ and study its properties. In Sect. 6 we apply a theorem of Uchiyama on the space $(X, \widetilde{d}, \mu)$ to complete the proof of Theorem 2. Finally, in Sect. 7 we provide some examples of semigroups that satisfy assumptions of Theorem 1 .

\section{Gaussian estimates and bounded harmonic functions}

In this section we assume that the semigroup $T_{t}$ satisfies (1.3). Clearly,

$$
C^{-1} \leq \int_{X} T_{t}(x, y) \mathrm{d} \mu(y) \leq C
$$

for all $t>0$ and a.e. $x \in X$. For a positive integer $n$ define

$$
\omega_{n}(x)=\frac{1}{n} \int_{0}^{n} \int_{X} T_{s}(x, y) \mathrm{d} \mu(y) \mathrm{d} s .
$$

Then,

$$
C^{-1} \leq \omega_{n}(x) \leq C .
$$

Recall that a metric space with the doubling condition is separable, then so is $L^{1}(X, \mu)$. Using the Banach-Alaoglu theorem for $L^{\infty}(X, \mu)$ there exists a subsequence $n_{k}$ and $\omega \in$ $L^{\infty}(X, \mu)$, such that $\omega_{n_{k}} \rightarrow \omega$ in $*$-weak topology. Obviously,

$$
C^{-1} \leq \omega(y) \leq C .
$$

Our goal is to prove that $T_{t} \omega(x)=\omega(x)$ for $t>0$. To this end, we write

$$
\begin{aligned}
\int_{X} T_{t}(x, y) \omega(y) \mathrm{d} \mu(y)= & \lim _{k \rightarrow \infty} \int_{X} T_{t}(x, y) \omega_{n_{k}}(y) \mathrm{d} \mu(y) \\
= & \lim _{k \rightarrow \infty} \frac{1}{n_{k}} \int_{0}^{n_{k}} \int_{X} T_{t+s}(x, z) \mathrm{d} \mu(z) \mathrm{d} s \\
= & \lim _{k \rightarrow \infty} \omega_{n_{k}}(x)+\lim _{k \rightarrow \infty} \frac{1}{n_{k}} \int_{n_{k}}^{n_{k}+t} \int_{X} T_{s}(x, z) \mathrm{d} \mu(z) \mathrm{d} s \\
& -\lim _{k \rightarrow \infty} \frac{1}{n_{k}} \int_{0}^{t} \int_{X} T_{s}(x, z) \mathrm{d} \mu(z) \mathrm{d} s .
\end{aligned}
$$

Since the last two limits tend to zero, as $k \rightarrow \infty$, we obtain

$$
\int_{X} T_{t}(x, y) \omega(y) \mathrm{d} \mu(y)=\lim _{k \rightarrow \infty} \omega_{n_{k}}(x) .
$$

From (2.1) we get that $\lim _{k \rightarrow \infty} \omega_{n_{k}}(x)$ exists for a.e. $x \in X$ and the limit has to be $\omega(x)$. Moreover, $T_{t} \omega(x)=\omega(x)$. Thus we have proved the following proposition.

Proposition 3 Assume that a semigroup $T_{t}$ satisfies (1.3). Then there exists a function $\omega$ and $C>0$ such that $0<C^{-1} \leq \omega(x) \leq C$ and $T_{t} \omega(x)=\omega(x)$ for every $t>0$. 


\section{Doob transform}

In this section we work in a slightly more general scheme. Let $(X, \mu)$ be a $\sigma$-finite measure space and $L$ be a self-adjoint operator on $L^{2}(X, \mu)$. We assume that the strongly continuous semigroup $T_{t}=\exp (-t L)$ admits a nonnegative integral kernel $T_{t}(x, y)$, so that

$$
T_{t} f(x)=\int_{X} T_{t}(x, y) f(y) \mathrm{d} \mu(y) .
$$

Moreover, assume that there exists $\omega$ satisfying $0<C^{-1} \leq \omega(x) \leq C$, that is $L$-harmonic. Namely, for every $t>0$ one has

$$
\int_{X} T_{t}(x, y) \omega(y) \mathrm{d} \mu(y)=\omega(x)
$$

for a.e. $x \in X$. Obviously, this implies that $\sup _{x \in X, t>0} \int T_{t}(x, y) \mathrm{d} \mu(y) \leq C$.

Define a new measure $\mathrm{d} \nu(x)=\omega^{2}(x) \mathrm{d} \mu(x)$ and a new kernel

$$
K_{t}(x, y)=\frac{T_{t}(x, y)}{\omega(x) \omega(y)} .
$$

The semigroup $K_{t}$ given by

$$
K_{t} f(x)=\int_{X} K_{t}(x, y) f(y) \mathrm{d} v(y)
$$

is a strongly continuous semigroup of self-adjoint integral operators on $L^{2}(X, v)$. The mapping $L^{2}(X, \mu) \ni f \mapsto \omega^{-1} f \in L^{2}(X, v)$ is an isometric isomorphism with the inverse $L^{2}(X, v) \ni g \mapsto \omega g \in L^{2}(X, \mu)$. Clearly,

$$
K_{t} g(x)=\omega(x)^{-1} T_{t}(\omega g)(x) .
$$

Moreover, the positive integral kernel $K_{t}(x, y)$ is conservative, that is,

$$
\int_{X} K_{t}(x, y) \mathrm{d} v(y)=\omega(x)^{-1} T_{t} \omega(x)=1 .
$$

Thus the above change of measure and operators, which is called Doob's transform (see, e.g., [16]), conjugates the semigroup $T_{t}$ with the conservative semigroup $K_{t}$.

It is clear that the operators $K_{t}$ are contractions on $L^{1}(X, v)$. Consequently, $K_{t}$ is a strongly continuous semigroup of linear operators on $L^{1}(X, v)$. To see this, it suffices to show that $\lim _{t \rightarrow 0}\left\|K_{t} \chi_{A}-\chi_{A}\right\|_{L^{1}(X, v)}=0$ for any measurable set $A$ of finite measure. We have that

$$
\lim _{t \rightarrow 0} \int_{A}\left|K_{t} \chi_{A}-\chi_{A}\right| \mathrm{d} v \leq \lim _{t \rightarrow 0}\left\|K_{t} \chi_{A}-\chi_{A}\right\|_{L^{2}(X, v)} v(A)^{1 / 2}=0 .
$$

On the other hand $\int_{X} K_{t} \chi_{A}(x) \mathrm{d} v(x)=v(A)=\left\|\chi_{A}\right\|_{L^{1}(X, v)}$. Hence,

$$
\begin{aligned}
\int_{A^{c}}\left|K_{t} \chi_{A}(x)\right| \mathrm{d} v(x) & =\int_{A^{c}} K_{t} \chi_{A}(x) \mathrm{d} v(x)=\int_{X} K_{t} \chi_{A}(x) \mathrm{d} v(x)-\int_{A} K_{t} \chi_{A}(x) \mathrm{d} v(x) \\
& =\int_{A} \chi_{A}(x) \mathrm{d} v(x)-\int_{A} K_{t} \chi_{A}(x) \mathrm{d} v(x) \rightarrow 0 \text { as } t \rightarrow 0,
\end{aligned}
$$

which completes the proof of the strong continuity of $K_{t}$ on $L^{1}(X, v)$. 
Further, we easily see that the semigroup $T_{t}$ is strongly continuous on $L^{1}(X, \mu)$. Indeed, if $f \in L^{1}(X, \mu)$ then $g=\omega^{-1} f \in L^{1}(X, v)$ and

$$
\begin{aligned}
\left\|T_{t} f-f\right\|_{L^{1}(X, \mu)} & =\int_{X}\left|K_{t} g(x)-g(x)\right| \omega(x)^{-1} \mathrm{~d} \nu(x) \\
& \lesssim \int_{X}\left|K_{t} g(x)-g(x)\right| \mathrm{d} \nu(x) \rightarrow 0 \text { as } t \rightarrow 0 .
\end{aligned}
$$

Now we discuss the equivalence of Theorems 1 and 2. Let $T_{t}$ and $K_{t}$ be the semigroups related by (3.1) with generators $-L$ and $-R$, respectively. It easily follows from the Doob transform that $f \in H^{1}(L)$ if and only if $\omega^{-1} f \in H^{1}(R)$ and $\|f\|_{H^{1}(L)} \simeq\left\|\omega^{-1} f\right\|_{H^{1}(R)}$. In other words

$$
H^{1}(L) \ni f \mapsto \omega^{-1} f \in H^{1}(R)
$$

is an isomorphism of the spaces.

Assume that the space $H^{1}(R)$ admits an atomic decomposition with atoms that satisfy the cancelation condition with respect to the measure $v$, that is every $g \in H^{1}(R)$ can be written as $g=\sum \lambda_{j} a_{j}$ with $\sum_{j}\left|\lambda_{j}\right| \simeq\|g\|_{H^{1}(R)}$ and $a_{j}$ are atoms with the property $\int a_{j} \mathrm{~d} v=0$. Then every $f \in H^{1}(L)$ admits atomic decomposition $f=\sum \lambda_{j} b_{j}$ with atoms $b_{j}$ that satisfy cancelation condition $\int b_{j} \omega \mathrm{d} \mu=0$.

\section{Hölder-type estimates on the semigroups}

In this section we consider a conservative semigroup $T_{t}$ having an integral kernel $T_{t}(x, y)$ that satisfies upper and lower Gaussian bounds (1.3). Let

$$
P_{t}(x, y)=\pi^{-1 / 2} \int_{0}^{\infty} e^{-u} T_{t^{2} /(4 u)}(x, y) \frac{d u}{\sqrt{u}}
$$

be the kernels of the subordinate semigroup $P_{t}=e^{-t \sqrt{L}}$.

Theorem 4 Let $L$ be a nonnegative self-adjoint linear operator on $L^{2}(X, \mu)$ such that the semigroup $T_{t}=e^{-t L}$ satisfies (1.3) and (1.6). Then there is a constant $\alpha>0$ such that

$$
\left|P_{t}(x, y)-P_{t}(x, z)\right| \lesssim\left(\frac{d(y, z)}{t}\right)^{\alpha} P_{t}(x, y)
$$

whenever $d(y, z) \leq t$.

Theorem 5 Under the assumptions of Theorem 4 there are constants $\alpha, c>0$ such that

$$
\left|T_{t}(x, y)-T_{t}(x, z)\right| \lesssim \mu(B(x, \sqrt{t}))^{-1}\left(\frac{d(y, z)}{\sqrt{t}}\right)^{\alpha} \exp \left(-\frac{c d(x, y)^{2}}{t}\right)
$$

whenever $d(y, z) \leq \sqrt{t}$.

Hölder regularity of semigroups satisfying Gaussian bounds was considered in various settings by many authors. We refer the reader to Grigor'yan [16], Hebisch and Saloff-Coste [19], Saloff-Coste [28], Gyrya and Saloff-Coste [17], Bernicot, Coulhon, and Frey [2], and references therein. Here we present a short alternative proof of (4.3). To this end we shall first prove some auxiliary propositions and then Theorem 4 . Finally, at the end of this section, we shall make use of functional calculi deducing Theorem 5 from Theorem 4. 
Proposition 6 For $x, y \in X$ and $t>0$ we have

$$
P_{t}(x, y) \simeq \frac{1}{\mu(B(x, t+d(x, y)))} \frac{t}{t+d(x, y)} .
$$

Proof Consider first the case $d(x, y) \leq t$. Then $d(x, y)+t \simeq t$. The upper bound follows by (1.3) and (1.1). Indeed,

$$
\begin{aligned}
P_{t}(x, y) & \lesssim \int_{0}^{1 / 4} \mu\left(B\left(x, \frac{t}{2 \sqrt{u}}\right)\right)^{-1} \frac{d u}{\sqrt{u}}+\mu(B(x, t))^{-1} \int_{1 / 4}^{\infty} \frac{e^{-u} \mu(B(x, t))}{\mu\left(B\left(x, \frac{t}{2 \sqrt{u}}\right)\right)} \frac{d u}{\sqrt{u}} \\
& \lesssim \mu(B(x, t))^{-1} \int_{0}^{1 / 4} \frac{d u}{\sqrt{u}}+\mu(B(x, t))^{-1} \int_{1 / 4}^{\infty} e^{-u}(2 \sqrt{u})^{q} \frac{d u}{\sqrt{u}} \\
& \lesssim \mu(B(x, t))^{-1}
\end{aligned}
$$

Also, (1.3) implies lower bounds, since

$$
P_{t}(x, y) \gtrsim \int_{1 / 4}^{1} \frac{e^{-u} \exp \left(-4 c_{1} u\right)}{\mu\left(B\left(x, \frac{t}{2 \sqrt{u}}\right)\right)} \frac{d u}{\sqrt{u}} \gtrsim \mu(B(x, t))^{-1}
$$

Let us now turn to the case $d(x, y) \geq t$. Then $d(x, y)+t \simeq d(x, y)$. Using (1.3), we have

$$
P_{t}(x, y) \lesssim \int_{0}^{\infty} \frac{e^{-u} \exp \left(-4 c_{2} u d(x, y)^{2} / t^{2}\right)}{\mu\left(B\left(x, \frac{t}{2 \sqrt{u}}\right)\right)} \frac{d u}{\sqrt{u}}=\int_{0}^{t^{2} / d(x, y)^{2}}+\int_{t^{2} / d(x, y)^{2}}^{\infty}=\left(J_{1}\right)+\left(J_{2}\right)
$$

Moreover, by (1.1),

$$
\begin{aligned}
\left(J_{1}\right) & \simeq \int_{0}^{t^{2} / d(x, y)^{2}} \frac{1}{\mu\left(B\left(x, \frac{t}{2 \sqrt{u}}\right)\right)} \frac{d u}{\sqrt{u}} \\
& \lesssim \int_{0}^{t^{2} / d(x, y)^{2}} \frac{1}{\mu\left(B\left(x, \frac{d(x, y)}{2}\right)\right)} \frac{d u}{\sqrt{u}} \simeq \frac{1}{\mu(B(x, d(x, y)))} \frac{t}{d(x, y)}
\end{aligned}
$$

and

$$
\begin{aligned}
\left(J_{2}\right) & \lesssim \frac{1}{\mu(B(x, d(x, y)))} \int_{t^{2} / d(x, y)^{2}}^{\infty} \exp \left(-4 c_{2} u d(x, y)^{2} / t^{2}\right)\left(\frac{2 d(x, y) \sqrt{u}}{t}\right)^{q} \frac{d u}{\sqrt{u}} \\
& \simeq \frac{1}{\mu(B(x, d(x, y)))} \frac{t}{d(x, y)}
\end{aligned}
$$

Estimates (4.5)-(4.7) give upper estimate. For the lower estimate, recall that $d(x, y) \geq t$ and observe that 


$$
\begin{aligned}
P_{t}(x, y) & \gtrsim \int_{t^{2} / d(x, y)^{2}}^{2 t^{2} / d(x, y)^{2}} \frac{e^{-u} \exp \left(-4 c_{1} u d(x, y)^{2} / t^{2}\right)}{\mu\left(B\left(x, \frac{t}{2 \sqrt{u}}\right)\right)} \frac{d u}{\sqrt{u}} \\
& \simeq \frac{1}{\mu(B(x, d(x, y) / 2))} \int_{t^{2} / d(x, y)^{2}}^{2 t^{2} / d(x, y)^{2}} \frac{d u}{\sqrt{u}} \\
& \simeq \frac{1}{\mu(B(x, d(x, y)))} \frac{t}{d(x, y)} .
\end{aligned}
$$

Corollary 7 There is a constant $C>0$ such that if $d(y, z) \leq t$, then

$$
C^{-1} \leq \frac{P_{t}(x, y)}{P_{t}(x, z)} \leq C .
$$

Proof The corollary is a simple consequence of (4.4). For the proof one may consider two cases: $d(x, y) \leq 2 t($ then $d(x, z) \leq 3 t)$ and $d(x, y)>2 t($ then $d(x, y) \simeq d(x, z))$.

Proposition 8 There exists a constant $\gamma \in(0,1)$ such that the following statement holds: If there are $y, z, t_{0}, a_{1}, b_{1}>0$ given such that $d(y, z)<t_{0}$ and for all $x \in X$ we have

$$
a_{1} \leq \frac{P_{t_{0}}(x, y)}{P_{t_{0}}(x, z)} \leq b_{1},
$$

then there is a subinterval $\left[a_{2}, b_{2}\right] \subseteq\left[a_{1}, b_{1}\right]$ such that $b_{2}-a_{2}=\gamma\left(b_{1}-a_{1}\right)$ and for all $t \geq 2 t_{0}$ and all $x \in X$ one has

$$
a_{2} \leq \frac{P_{t}(x, y)}{P_{t}(x, z)} \leq b_{2}
$$

Proof The proof, which takes some ideas from [21], is an adapted version of the proof of [13, Prop. 3.1]. For the reader convenience we present the details. Let $m=\left(a_{1}+b_{1}\right) / 2$ and $\theta=\left(b_{1}-a_{1}\right) /\left(a_{1}+b_{1}\right) \in(0,1)$, so that $a_{1}=(1-\theta) m$ and $b_{1}=(1+\theta) m$. Define

$$
\Omega_{+}=\left\{x \in X \mid m \leq \frac{P_{t_{0}}(x, y)}{P_{t_{0}}(x, z)} \leq b_{1}\right\}, \quad \Omega_{-}=X \backslash \Omega_{+} .
$$

Obviously, either $\mu\left(\Omega_{-} \cap B\left(z, t_{0}\right)\right) \geq \mu\left(B\left(z, t_{0}\right)\right) / 2$ or $\mu\left(\Omega_{+} \cap B\left(z, t_{0}\right)\right) \geq \mu\left(B\left(z, t_{0}\right)\right) / 2$. Here we shall assume that the latter holds. The proof in the opposite case is similar. Denote $B=B\left(z, t_{0}\right), s=t-t_{0} \geq t_{0}$. For $x \in X$, we have

$$
\begin{aligned}
P_{t}(x, y) & \geq m(1-\theta) \int_{\Omega_{-}} P_{s}(x, w) P_{t_{0}}(w, z) \mathrm{d} \mu(w)+m \int_{\Omega_{+}} P_{S}(x, w) P_{t_{0}}(w, z) \mathrm{d} \mu(w) \\
& =m(1-\theta) P_{t}(x, z)+m \theta \int_{\Omega_{+}} P_{s}(x, w) P_{t_{0}}(w, z) \mathrm{d} \mu(w) \\
& \geq m(1-\theta) P_{t}(x, z)+m \theta \int_{\Omega_{+} \cap B} P_{s}(x, w) P_{t_{0}}(w, z) \mathrm{d} \mu(w) \\
& \geq m(1-\theta) P_{t}(x, z)+m \theta \frac{\mu(B)}{2} \inf _{w \in B} P_{s}(x, w) \inf _{w \in B} P_{t_{0}}(w, z)=(J) .
\end{aligned}
$$

Notice that $d(w, z) \leq t_{0} \leq s$ and, by Corollary 7 ,

$$
\inf _{w \in B} P_{S}(x, w) \simeq P_{S}(x, z) .
$$


Since $t \simeq s$, Proposition 6 implies

$$
P_{S}(x, z) \simeq P_{t}(x, z)
$$

and

$$
\inf _{w \in B} P_{t_{0}}(w, z) \simeq \mu(B)^{-1}
$$

Therefore

$$
(J) \geq m((1-\theta)+\kappa \theta) P_{t}(x, z)=m(1-\theta(1-\kappa)) P_{t}(x, z),
$$

where $\kappa \in(0,2)$. Moreover, from (4.9) and the semigroup property we easily get

$$
P_{t}(x, y) \leq b_{1} \int P_{s}(x, w) P_{t_{0}}(w, z) \mathrm{d} \mu(w) \leq b_{1} P_{t}(x, z) .
$$

Defining $\gamma=1-\kappa / 2 \in(0,1), b_{2}=b_{1}$ and $a_{2}=m((1-\theta)+\kappa \theta)$, we have

$$
b_{2}-a_{2}=2 m \theta(1-\kappa / 2)=\left(b_{1}-a_{1}\right)(1-\kappa / 2)=\gamma\left(b_{1}-a_{1}\right) .
$$

Now (4.10) together with (4.11) gives

$$
a_{2} \leq \frac{P_{t}(x, y)}{P_{t}(x, z)} \leq b_{2} \text { for } x \in X
$$

Proof of Thorem 4 Having Corollary 7 and Proposition 8 proved, we follow arguments of [13] to obtain the theorem. By Corollary 7 there are $b_{1}>a_{1}>0$ such that for $y, z \in X$ and $t>0$ satisfying $d(y, z)<t$ we have

$$
a_{1} \leq \frac{P_{t}(x, y)}{P_{t}(x, z)} \leq b_{1}
$$

for all $x \in X$. From Proposition 8 we deduce that there exists $\omega(y, z)$ such that

$$
\lim _{t \rightarrow \infty} \frac{P_{t}(x, y)}{P_{t}(x, z)}=\omega(y, z) \quad \text { uniformly in } x \in X .
$$

It follows from (4.1) that $\int_{X} P_{t}(x, y) \mathrm{d} \mu(y)=1$. Recall that $P_{t}(x, y)=P_{t}(y, x)$. Using (4.12),

$$
1=\int P_{t}(y, x) \mathrm{d} \mu(x)=\int \frac{P_{t}(y, x)}{P_{t}(z, x)} P_{t}(z, x) \mathrm{d} \mu(x) \underset{t \rightarrow \infty}{\longrightarrow} \omega(y, z) .
$$

Thus $\omega(y, z)=1$.

Assume that $d(y, z)<t$. Let $n \in \mathbb{N}$ be such that $d(y, z) \leq t 2^{-n}<2 d(y, z)$. Set $t_{0}=t 2^{-n}$. Clearly, $d(y, z) \leq t_{0}$ and

$$
a_{1} \leq \frac{P_{t_{0}}(x, y)}{P_{t_{0}}(x, z)} \leq b_{1} .
$$

Observe that $n \simeq \log (t / d(y, z))$. Applying Proposition $8 n$-times we arrive at

$$
\left|\frac{P_{t}(x, y)}{P_{t}(x, z)}-1\right| \lesssim \gamma^{n} \lesssim \gamma^{c \log (t / d(y, z))} \lesssim\left(\frac{d(y, z)}{t}\right)^{\alpha},
$$

with $\alpha>0$ and the proof of Theorem 4 is finished. 
Finally, we devote the remaining part of this section for deducing Theorem 5 from Theorem 4. This is done by using a functional calculi. First, we need some preparatory facts. Recall that $q$ is a fixed constant satisfying (1.1). By $W^{2, \sigma}(\mathbb{R})$ we denote the Sobolev space with the norm

$$
\|f\|_{W^{2, \sigma}(\mathbb{R})}=\left(\int_{\mathbb{R}}\left(1+|\xi|^{2}\right)^{\sigma}|\widehat{f}(\xi)|^{2} \mathrm{~d} \xi\right)^{1 / 2} .
$$

Let $\int_{0}^{\infty} \xi \mathrm{d} E_{\sqrt{L}}(\xi)$ be the spectral resolution for $\sqrt{L}$. For a bounded function $m$ on $[0, \infty)$ the formula

$$
m(\sqrt{L})=\int_{0}^{\infty} m(\xi) \mathrm{d} E_{\sqrt{L}}(\xi)
$$

defines a bounded linear operator on $L^{2}(X, \mu)$.

Further we shall use the following lemma, whose proof based on finite speed propagation of the wave equation (see [9]) can be found in [11].

Lemma 9 [11, Lemma 4.8] Let $\kappa>1 / 2, \beta>0$. Then there exists a constant $C>0$ such that for every even function $m \in W^{2, \beta / 2+\kappa}(\mathbb{R})$ and every $g \in L^{2}(X, \mu)$, supp $g \subset B\left(y_{0}, r\right)$, we have

$\int_{d\left(x, y_{0}\right)>2 r}\left|m\left(2^{-j} \sqrt{L}\right) g(x)\right|^{2}\left(\frac{d\left(x, y_{0}\right)}{r}\right)^{\beta} d \mu(x) \leq C\left(r 2^{j}\right)^{-\beta}\|m\|_{W^{2, \beta / 2+\kappa}(\mathbb{R})}^{2}\|g\|_{L^{2}(X, \mu)}^{2}$

for $j \in \mathbb{Z}$.

Proposition 10 Let $\beta>q$ and $\kappa>1 / 2$. There is a constant $C>0$ such that for every $F \in W^{2, \beta / 2+\kappa}(\mathbb{R})$ with supp $F \subset(1 / 2,2)$ the integral kernels $F\left(2^{-j} \sqrt{L}\right)(x, y)$ of the operators $F\left(2^{-j} \sqrt{L}\right)$ satisfy

$$
\int_{X}\left|F\left(2^{-j} \sqrt{L}\right)(x, y)\right| d \mu(x) \leq C\|F\|_{W^{2, \beta / 2+\kappa}(\mathbb{R})} \text { for } j \in \mathbb{Z} .
$$

Proof For $y \in X$ and $j \in \mathbb{Z}$ set $U_{0}=B\left(y, 2^{-j}\right), U_{k}=B\left(y, 2^{k-j}\right) \backslash B\left(y, 2^{k-j-1}\right)$, $k=1,2, \ldots$ Define $g_{j, k, y}(x)=T_{2^{-2 j}}(x, y) \chi_{U_{k}}(x), k=0,1,2, \ldots$ Then, using (1.1) and (1.3), we have

$$
\left\|g_{j, y, k}\right\|_{L^{2}(X, \mu)} \leq a_{k} \mu\left(U_{0}\right)^{-1 / 2}
$$

where $a_{k}=C_{0} \sqrt{C_{q} 2^{k q}} \exp \left(-c_{2} 2^{2 k-2}\right)$ is a rapidly decreasing sequence.

Let $m(\xi)$ be the even extension of $e^{\xi^{2}} F(\xi)$. Obviously, $\|m\|_{W^{2, \beta / 2+\kappa}(\mathbb{R})} \simeq\|F\|_{W^{2, \beta / 2+\kappa}(\mathbb{R})}$. Then, $F\left(2^{-j} \sqrt{L}\right)=m\left(2^{-j} \sqrt{L}\right) T_{2^{-2 j}}$, and, consequently,

$$
F\left(2^{-j} \sqrt{L}\right)(x, y)=\sum_{k=0}^{\infty} m\left(2^{-j} \sqrt{L}\right) g_{j, y, k}(x) .
$$

By the Cauchy-Schwartz inequality, (1.1), and (4.13) we get

$$
\begin{aligned}
\| m & \left(2^{-j} \sqrt{L}\right) g_{j, y, k}\left\|_{L^{1}\left(B\left(y, 2^{k-j+1}\right), \mu\right)} \leq \mu\left(B\left(y, 2^{k-j+1}\right)\right)^{1 / 2}\right\| m\left(2^{-j} \sqrt{L}\right) g_{j, y, k} \|_{L^{2}(X, \mu)} \\
& \lesssim\left(\frac{\mu\left(B\left(y, 2^{k-j+1}\right)\right)}{\mu\left(B\left(y, 2^{-j}\right)\right)}\right)^{1 / 2} \mu\left(B\left(y, 2^{-j}\right)\right)^{1 / 2}\|m\|_{L^{\infty}(\mathbb{R})}\left\|g_{j, y, k}\right\|_{L^{2}(X, \mu)} \\
& \lesssim 2^{k q} a_{k}\|m\|_{W^{2, \beta / 2+\kappa}(\mathbb{R})} .
\end{aligned}
$$


We turn to estimate $\left\|m\left(2^{-j} \sqrt{L}\right) g_{j, y, k}\right\|_{L^{1}\left(B\left(y, 2^{k-j+1}\right)^{c}, \mu\right)}$. Utilizing the Cauchy-Schwarz inequality and Lemma 9, we obtain,

$$
\begin{aligned}
& \int_{d(x, y)>2^{k-j+1}}\left|m\left(2^{-j} \sqrt{L}\right) g_{j, y, k}(x)\right| \mathrm{d} \mu(x) \\
& \leq\left[\int_{d(x, y)>2^{k-j+1}}\left|m\left(2^{-j} \sqrt{L}\right) g_{j, y, k}(x)\right|^{2}\left(\frac{d(x, y)}{2^{k-j}}\right)^{\beta} \mathrm{d} \mu(x)\right]^{1 / 2} \\
& \quad \times\left[\int_{d(x, y)>2^{k-j+1}}\left(\frac{d(x, y)}{2^{k-j}}\right)^{-\beta} \mathrm{d} \mu(x)\right]^{1 / 2} \\
& \lesssim 2^{-k \beta / 2}\|m\|_{W^{2, \beta / 2+\kappa}(\mathbb{R})}\left\|g_{j, y, k}\right\|_{L^{2}(X, \mu)}\left[\int_{d(x, y)>2^{k-j+1}}\left(\frac{d(x, y)}{2^{k-j}}\right)^{-\beta} \mathrm{d} \mu(x)\right]^{1 / 2} .
\end{aligned}
$$

Recall that $\beta>q$ and hence is not difficult to check that (1.1) leads to

$$
\int_{d(x, y)>2^{k-j+1}}\left(\frac{d(x, y)}{2^{k-j}}\right)^{-\beta} \mathrm{d} \mu(x) \lesssim 2^{k q} \mu\left(U_{0}\right) .
$$

Thus, by (4.13), (4.16), and (4.17), we get

$$
\int_{d(x, y)>2^{k-j+1}}\left|m\left(2^{-j} \sqrt{L}\right) g_{j, y, k}(x)\right| \mathrm{d} \mu(x) \lesssim 2^{-k \beta / 2} 2^{k q / 2}\|m\|_{W^{2, \beta / 2+\kappa}(\mathbb{R})} a_{k},
$$

which, combined with (4.15) and (4.14), completes the proof of the proposition.

For $t>0$ set

$$
\psi_{t}(\xi)=\exp \left(\sqrt{t} \xi-t \xi^{2}\right)
$$

Lemma 11 The operators $\psi_{t}(\sqrt{L})$ have integral kernels $\Psi_{t}(x, y)$ that satisfy

$$
\sup _{x \in X, t>0} \int\left|\Psi_{t}(x, y)\right| d \mu(y) \leq C .
$$

Proof Denoting $\theta_{t}(\xi)=\exp \left(-t \xi^{2}\right)(\exp (\sqrt{t} \xi)-1)$, where $t, \xi>0$, we have

$$
\psi_{t}(\sqrt{L})=T_{t}+\theta_{t}(\sqrt{L}) .
$$

Clearly, by (1.3), $\sup _{t, x>0} \int T_{t}(x, y) \mathrm{d} \mu(y) \leq C$. Thus we concentrate our attention on $\theta_{t}(\sqrt{L})$.

Let $\eta \in C_{c}^{\infty}(1 / 2,2)$ be a partition of unity such that

$$
\theta_{t}(\xi)=\sum_{j \in \mathbb{Z}} \theta_{t}(\xi) \eta\left(2^{-j} \xi\right)=\sum_{j \in \mathbb{Z}} \theta_{t, j}(\xi)
$$

Denote $\widetilde{\theta_{t, j}}(\xi)=\theta_{t, j}\left(2^{j} \xi\right)=\eta(\xi) \theta_{1}\left(\sqrt{t} 2^{j} \xi\right)$. One can easily verify that for $n \in \mathbb{N} \cup\{0\}$ there are constants $C_{n}, c_{n}>0$ such that

$$
\left\|\frac{d^{n}}{\mathrm{~d} \xi^{n}} \widetilde{\theta}_{t, j}\right\|_{L^{\infty}(\mathbb{R})} \leq C_{n}\left\{\begin{array}{ll}
\sqrt{t} 2^{j} & \text { for } j \leq-\log _{2} \sqrt{t} \\
\exp \left(-c_{n} t 2^{2 j}\right) & \text { for } j \geq-\log _{2} \sqrt{t}
\end{array} .\right.
$$


In other words, for arbitrary $N$,

$$
\sum_{j \in \mathbb{Z}}\left\|\widetilde{\theta}_{t, j}\right\|_{W^{2, N}(\mathbb{R})} \leq C(N)
$$

Using Proposition 10 (with a fixed $N>q / 2+1$ ) we get that the integral kernels $\Theta_{t, j}(x, y)$ of the operators $\theta_{t, j}(\sqrt{L})=\widetilde{\theta}_{t, j}\left(2^{-j} \sqrt{L}\right)$ satisfy

$$
\int\left|\Theta_{t, j}(x, y)\right| \mathrm{d} \mu(y) \lesssim\left\|\widetilde{\theta}_{t, j}\right\|_{W^{2, N}(\mathbb{R})}
$$

Therefore, $\Theta_{t}(x, y)=\sum_{j \in \mathbb{Z}} \Theta_{t, j}(x, y)$ is the integral kernel of $\theta_{t}(\sqrt{L})$ and it satisfies

$$
\sup _{t, x>0} \int\left|\Theta_{t}(x, y)\right| \mathrm{d} \mu(y) \leq C .
$$

Proof of Theorem 5 By the spectral theorem $T_{t}=\psi_{t}(\sqrt{L}) P_{\sqrt{t}}$ and

$$
T_{t}(x, y)=\int \Psi_{t}(x, w) P_{\sqrt{t}}(w, y) \mathrm{d} \mu(w) .
$$

Using Theorem 4 together with Lemma 11 and Proposition 6 , for $\sqrt{t} \geq d(y, z)$, we have

$$
\begin{aligned}
\left|T_{t}(x, y)-T_{t}(x, z)\right| & =\left|\int \Psi_{t}(x, w)\left(P_{\sqrt{t}}(w, y)-P_{\sqrt{t}}(w, z)\right) \mathrm{d} \mu(w)\right| \\
& \lesssim\left(\frac{d(y, z)}{\sqrt{t}}\right)^{\alpha} \int\left|\Psi_{t}(x, w)\right| P_{\sqrt{t}}(w, y) \mathrm{d} \mu(w) \\
& \lesssim \mu(B(y, \sqrt{t}))^{-1}\left(\frac{d(y, z)}{\sqrt{t}}\right)^{\alpha} .
\end{aligned}
$$

We claim that for $d(y, z) \leq \sqrt{t}$ one has

$$
\left|T_{t}(x, y)-T_{t}(x, z)\right| \lesssim \mu(B(y, \sqrt{t}))^{-1}\left(\frac{d(y, z)}{\sqrt{t}}\right)^{\alpha / 2} \exp \left(-\frac{c d(x, y)^{2}}{t}\right) .
$$

To prove the claim we consider two cases.

Case 1: $2 d(y, z) \geq d(x, y)$. Recall that $\sqrt{t} \geq d(y, z)$; thus, $d(x, y) \leq 2 \sqrt{t}$ and (4.19) follows directly from (4.18).

Case 2: $2 d(y, z) \leq d(x, y)$. In this case $d(x, y) \simeq d(x, z)$, so by (1.3) we obtain

$$
\begin{aligned}
\left|T_{t}(x, y)-T_{t}(x, z)\right| & \lesssim \frac{\exp \left(-\frac{c_{2} d(x, y)^{2}}{t}\right)}{\mu(B(y, \sqrt{t}))}+\frac{\exp \left(-\frac{c_{2} d(x, z)^{2}}{t}\right)}{\mu(B(z, \sqrt{t}))} \\
& \lesssim \mu(B(y, \sqrt{t}))^{-1} \exp \left(-\frac{c d(x, y)^{2}}{t}\right),
\end{aligned}
$$

where in the last inequality we have utilized that $\mu(B(z, \sqrt{t})) \simeq \mu(B(y, \sqrt{t}))$, since $d(y, z)<\sqrt{t}$. By taking the geometric mean of (4.18) and (4.20) we obtain (4.19). To finish the proof observe that (4.19) implies (4.3). Indeed,

$$
\mu(B(x, \sqrt{t}))=\mu(B(y, \sqrt{t})) \frac{\mu(B(x, \sqrt{t}))}{\mu(B(y, \sqrt{t}))} \leq \mu(B(y, \sqrt{t})) \frac{\mu(B(y, \sqrt{t}+d(x, y)))}{\mu(B(y, \sqrt{t}))}
$$




$$
\leq \mu(B(y, \sqrt{t}))\left(1+\frac{d(x, y)}{\sqrt{t}}\right)^{q}
$$

and using the exponent factor we can replace $\mu(B(y, \sqrt{t}))$ by $\mu(B(x, \sqrt{t}))$.

Remark 12 Let us remark that Lemma 11, which is crucial in our proof of Theorem 5, can be proved by applying functional calculus of Hebisch [18]. Thus, Theorem 5 can be also obtained without using the finite propagation speed of the solution of the wave equation

$$
\left(\partial_{t}^{2}+L\right) u(t, x)=0, u(0, x)=u_{0}(x), \partial_{t} u(0, x)=0 .
$$

As a consequence of (1.4) and Theorem 5 we get what follows.

Corollary 13 The function $T_{t}(x, y)$ is continuous on $(0, \infty) \times X \times X$.

As a direct consequence of Theorem 5 and Doob transform (see (3.1)) we get the following corollary. Notice that in Corollary 14 we do not assume that $T_{t}(x, y)$ is conservative.

Corollary 14 Assume that the semigroup $T_{t}$ satisfies (1.3). Then there are constants $\alpha, c>0$ such that

$$
\left|\frac{T_{t}(x, y)}{\omega(x) \omega(y)}-\frac{T_{t}(x, z)}{\omega(x) \omega(z)}\right| \lesssim \mu(B(x, \sqrt{t}))^{-1}\left(\frac{d(y, z)}{\sqrt{t}}\right)^{\alpha} \exp \left(-\frac{c d(x, y)^{2}}{t}\right)
$$

whenever $d(y, z) \leq \sqrt{t}$.

\section{Measures and distances}

To prove Theorem 2 we introduce a new quasi-metric $\widetilde{d}$ on $X$, which is related to $d$ and $\mu$. To this end, set

$$
\widetilde{d}(x, y)=\inf \mu(B),
$$

where the infimum is taken over all closed balls $B$ containing $x$ and $y$ (see, e.g., $[8,25]$ ). Denote

$$
\widetilde{B}(x, r)=\{y \in X \mid \widetilde{d}(x, y) \leq r\} .
$$

In the lemma below we state some properties of $\tilde{d}$, which are known among specialists, and which we shall need latter on. Since their proofs are very short and it is difficult for us to indicate one reference which contains all of them, we provide the details for the convenience of the reader.

Lemma 15 The function $\widetilde{d}$ has the following properties: 0

(a) there exists $C_{b}>0$ such that for $x, y \in X$ we have

$$
C_{b}^{-1} \mu(B(x, d(x, y))) \leq \widetilde{d}(x, y) \leq \mu(B(x, d(x, y))) .
$$

(b) $\widetilde{d}$ is a quasi-metric, namely there exists $A_{1}$ such that

$$
\tilde{d}(x, y) \leq A_{1}(\widetilde{d}(x, z)+\widetilde{d}(z, y)) .
$$

Moreover, if the measure $\mu$ has no atoms and $\mu(X)=\infty$, then:

(c) the measure $\mu$ is regular with respect to $\widetilde{d}$, namely for $x \in X$ and $r>0$,

$$
\mu(\widetilde{B}(x, r)) \simeq r
$$


(d) for $x \in X$ and $r>0$ there exists $R>0$ such that

$$
\widetilde{B}(x, r) \subset B(x, R) \text { and } \mu(B(x, R)) \lesssim \mu(\widetilde{B}(x, r)) ;
$$

(e) for $x \in X$ and $R>0$ there exists $r>0$ such that

$$
B(x, R) \subset \widetilde{B}(x, r) \text { and } \mu(\widetilde{B}(x, r)) \lesssim \mu(B(x, R)) .
$$

Proof (a) Set $R=d(x, y)$. Clearly, $\tilde{d}(x, y) \leq \mu(B(x, R))$, as $x$ and $y$ belong to $B(x, R)$. On the other hand, if $x$ and $y$ belong to a ball $B=B(z, r)$, then $R \leq 2 r$; hence, $B(x, R) \subset$ $B(z, 3 r)$ and $\mu(B(x, R)) \leq \mu(B(z, 3 r)) \simeq \mu(B(z, r))$. By taking the infimum over all balls $B$ containing both $x$ and $y$, we conclude that $\mu(B(x, R)) \leq C \widetilde{d}(x, y)$.

(b) For every $x, y, z \in X$, we have $d(x, y) \leq d(x, z)+d(z, y)$. Assume that $r=d(x, z) \geq$ $d(z, y)$. Then $x, y \in B(z, r)$. By using (a), we deduce that

$$
\widetilde{d}(x, y) \leq \mu(B(z, r)) \simeq \widetilde{d}(z, x) \leq \widetilde{d}(x, z)+\widetilde{d}(z, y) .
$$

(c) Given $x \in X$, by our additional assumptions, the function $(0, \infty) \ni r \mapsto \mu(B(x, r))$ is increasing and

$$
\begin{cases}\mu(B(x, r)) \searrow 0 & \text { as } r \searrow 0, \\ \mu(B(x, r)) \nearrow+\infty & \text { as } r \nearrow+\infty .\end{cases}
$$

Let $x \in X$ and $r>0$. For every $y \in \widetilde{B}(x, r)$, we have $\mu(B(x, d(x, y)) \simeq \widetilde{d}(x, y) \leq r$. Hence

$$
R=\sup \{d(x, y) \mid y \in \widetilde{B}(x, r)\}<+\infty .
$$

Let $y \in \widetilde{B}(x, r)$ such that $d(x, y) \geq \frac{R}{2}$. Then

$$
\widetilde{B}(x, r) \subset B(x, R) \subset B(x, 2 d(x, y)) .
$$

Hence

$$
\mu(\widetilde{B}(x, r)) \leq \mu(B(x, R)) \leq \mu(B(x, 2 d(x, y))) \simeq \mu(B(x, d(x, y))) \simeq \widetilde{d}(x, y) \leq r .
$$

On the other hand,

$$
T=\inf \{t>0 \mid \mu(B(x, t)) \geq r\}>0 .
$$

$\underset{\sim}{\operatorname{As}} \mu(B(x, T / 2))<r$, we have $\widetilde{d}(x, y)<r$, for every $y \in B(x, T / 2)$; hence, $B(x, T / 2) \subset$ $\widetilde{B}(x, r)$. Consequently,

$$
r \leq \mu(B(x, 2 T)) \simeq \mu(B(x, T / 2)) \leq \mu(\widetilde{B}(x, r)),
$$

which together with (5.3) completes the proof of (c).

(d) is a simple consequence of (5.2), (5.3), and (c).

(e) Set $r=\mu(B(x, R))$. If $y \in B(x, R)$, then $\widetilde{d}(x, y) \leq r$ and, consequently, $B(x, R) \subset$ $\widetilde{B}(x, r)$. Clearly, by $(\mathrm{c}), \mu(\widetilde{B}(x, r)) \simeq r=\mu(B(x, R))$.

Let us recall that in Theorems 1 and 2 we assume that $\Phi_{x}$ is a bijection on $(0, \infty)$. This obviously implies that $\mu(X)=\infty$ and that $\mu$ is nonatomic. As a consequence of (d) and (e) of Lemma 15 we obtain the following corollary.

Corollary 16 Suppose that $\mu$ has no atoms and $\mu(X)=\infty$. Then, the atomic Hardy spaces $H_{\mathrm{at}}^{1}(d, \mu)$ and $H_{\mathrm{at}}^{1}(\widetilde{d}, \mu)$ coincide and the corresponding atomic norms are equivalent.

We finish this section by Lemma 17 , which is used latter on. Define $A_{2}:=C_{b}\left(C_{d} 2^{q}\right)^{3}$, where $C_{d}, q$, and $C_{b}$ are as in (1.1) and (5.1). 
Lemma 17 Suppose that we have a space of homogeneous type $(X, d, \mu)$ such that the function $\Phi_{x}$ defined in (1.5) is a bijection on $(0, \infty)$. Assume that $x \in X, r, t>0$ are related by $r=\mu(B(x, \sqrt{t}))$ and satisfy: $\sqrt{t} \leq d(y, z), A_{2} \widetilde{d}(y, z)<r$. Then

$$
\sqrt{t} \leq d(x, y) \leq 2 d(x, z) .
$$

Proof Suppose, toward a contradiction, that $d(x, y)<\sqrt{t}$. From (5.1) we get

$$
\begin{aligned}
r & =\mu(B(x, \sqrt{t})) \leq \mu(B(y, 2 \sqrt{t})) \leq C_{d} 2^{q} \mu(B(y, \sqrt{t})) \\
& \leq C_{d} 2^{q} \mu(B(y, d(y, z))) \leq C_{d} 2^{q} C_{b} \widetilde{d}(y, z)<r,
\end{aligned}
$$

so the first inequality is proved.

Similarly, assume $d(x, z)<d(x, y) / 2$. Then $d(x, y) / 2 \leq d(y, z) \leq 2 d(x, y)$. Thus, using (5.1),

$$
\begin{aligned}
\tilde{d}(y, z) & \geq C_{b}^{-1} \mu\left(B(y, d(y, z)) \geq C_{b}^{-1}\left(C_{d} 2^{q}\right)^{-1} \mu(B(y, d(x, y))\right. \\
& \geq C_{b}^{-1}\left(C_{d} 2^{q}\right)^{-2} \mu(B(x, d(x, y))) \geq C_{b}^{-1}\left(C_{d} 2^{q}\right)^{-3} \mu(B(x, \sqrt{t}))=A_{2}^{-1} r
\end{aligned}
$$

and we come to a contradiction.

\section{Proof of Theorem 2}

In order to prove Theorem 2 we shall use a result of Uchiyama [33], which we state below in Theorem 18 . Denote by $(X, \tilde{d}, \mu)$ the space $X$ equipped with a quasi-metric $\widetilde{d}$ and a nonnegative measure $\mu$, where $\mu(X)=\infty$. Assume moreover that

$$
\mu(\widetilde{B}(x, r)) \simeq r
$$

where $x \in X, r>0$ and $\widetilde{B}(x, r) \subseteq X$ is a ball in the quasi-metric $\widetilde{d}$. Let $A_{1}$ be a constant in the quasi-triangle inequality, i.e.,

$$
\tilde{d}(x, y) \leq A_{1}(\tilde{d}(x, z)+\widetilde{d}(z, y)), \quad x, y, z \in X .
$$

Additionally, we impose that there exist constants $\gamma_{1}, \gamma_{2}, \gamma_{3}>0, A \geq A_{1}$ and a continuous function $\widetilde{T}(r, x, y)$ of variables $x, y \in X$ and $r>0$ such that

$$
\begin{aligned}
& \widetilde{T}(r, x, x) \gtrsim r^{-1}, \\
& 0 \leq \widetilde{T}(r, x, y) \lesssim r^{-1}\left(1+\frac{\tilde{d}(x, y)}{r}\right)^{-1-\gamma_{1}}, \\
& \text { if } \widetilde{d}(y, z)<(r+\widetilde{d}(x, y)) /(4 A), \text { then } \\
& |\widetilde{T}(r, x, y)-\widetilde{T}(r, x, z)| \lesssim r^{-1}\left(\frac{\tilde{d}(y, z)}{r}\right)^{\gamma_{2}}\left(1+\frac{\tilde{d}(x, y)}{r}\right)^{-1-\gamma_{3}},
\end{aligned}
$$

for all $x, y, z \in X$ and $r>0$.

As in [33], we consider the maximal function

$$
f^{(+)}(x)=\sup _{r>0}\left|\int_{X} \tilde{T}(r, x, y) f(y) \mathrm{d} \mu(y)\right|
$$

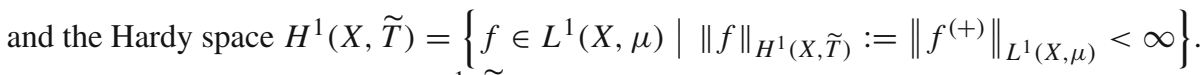

Recall that the atomic space $H_{\text {at }}^{1}(\tilde{d}, \mu)$ is defined in Sect. 1. 
Theorem 18 ([33], Corollary 1') Suppose that (X, $\widetilde{d}, \mu, \widetilde{T})$ satisfy (6.1), (6.2), (U1), (U2), and (U3). Then the spaces $H^{1}(X, \widetilde{T})$ and $H_{\mathrm{at}}^{1}(\widetilde{d}, \mu)$ coincide and

$$
\|f\|_{H^{1}(X, \widetilde{T})} \simeq\|f\|_{H_{\mathrm{at}}^{1}(\widetilde{d}, \mu)} .
$$

Assume that the kernel $T_{t}(x, y)$ satisfies (1.3) and the semigroup $T_{t}$ is conservative. Recall that in Sect. 4 we proved Hölder-type estimate (4.3) for $T_{t}(x, y)$. Define $\widetilde{T}(r, x, y)$ by

$$
\widetilde{T}(r, x, y):=T_{t}(x, y),
$$

where $t=t(x, r)$ is such that

$$
\mu(B(x, \sqrt{t}))=r .
$$

In what follows $t, r>0$ and $x \in X$ are always related by (6.3). Let us notice that from Corollary 13 and by the assumption that $\Phi_{x}$ is a continuous bijection on $(0, \infty)$ we have that $\widetilde{T}$ is a continuous function on $(0, \infty) \times X \times X$.

Theorem 19 Suppose that $T_{t}(x, y)$ satisfies upper and lower Gaussian bounds (1.3) and the semigroup $T_{t}$ is conservative. Then the kernel $\widetilde{T}(r, x, y)$ satisfies (U1), (U2), and (U3).

Proof On-diagonal lower estimate (U1) is an immediate consequence of lower Gaussian bound (1.3).

For every fixed $\delta>0$, the upper estimate

$$
\widetilde{T}(r, x, y) \lesssim r^{-1}\left(1+\frac{\widetilde{d}(x, y)}{r}\right)^{-1-\delta}
$$

follows from the upper estimates for $T_{t}(x, y)$, more precisely by combining

$$
\widetilde{T}(r, x, y) \lesssim r^{-1} \exp \left(-\frac{c d(x, y)^{2}}{t}\right)
$$

with

$$
\begin{aligned}
\left(1+\frac{\tilde{d}(x, y)}{r}\right)^{1+\delta} & \leq\left(1+\frac{\mu(B(x, d(x, y)))}{\mu(B(x, \sqrt{t}))}\right)^{1+\delta} \\
& \lesssim\left(1+\frac{d(x, y)}{\sqrt{t}}\right)^{q(1+\delta)} \lesssim \exp \left(\frac{c d(x, y)^{2}}{t}\right)
\end{aligned}
$$

The latter estimate holds for any $\delta>0$. Thus (U2) is proved with any $\gamma_{1}>0$. To finish the proof we need Hölder-type estimate (U3). This is proved in Proposition 20 below.

Proposition 20 Let $\alpha$ be a constant as in Theorem 5. There exists $A \geq A_{1}$ such that for $\delta>0$ we have

$$
|\widetilde{T}(r, x, y)-\widetilde{T}(r, x, z)| \lesssim r^{-1}\left(1+\frac{\tilde{d}(x, y)}{r}\right)^{-1-\delta}\left(\frac{\tilde{d}(y, z)}{r}\right)^{\frac{\alpha}{q}}
$$

if $\widetilde{d}(y, z) \leq(r+\widetilde{d}(x, y)) /(4 A)$.

Proof Set $A=\max \left(A_{1}, A_{2}\right)$, see (6.2) and Lemma 17. Assuming that $\widetilde{d}(y, z) \leq(r+$ $\widetilde{d}(x, y)) /(4 A)$ let us begin with some observations.

Firstly, we claim that it suffices to prove (6.7) for $\widetilde{d}(y, z)<r /(2 A)$. Indeed, if $\widetilde{d}(y, z)>$ $r /(2 A)$, then $\tilde{d}(y, z) \leq \tilde{d}(x, y) /(2 A)$ and, consequently,

$$
\widetilde{d}(x, y) \leq A_{1} \widetilde{d}(x, z)+A_{1} \widetilde{d}(z, y) \leq A_{1} \widetilde{d}(x, z)+\widetilde{d}(x, y) / 2 .
$$


So, $\widetilde{d}(x, y) \lesssim \widetilde{d}(x, z)$ and (6.7) follows from (6.4) by using the triangle inequality. From now on we assume that $\widetilde{d}(y, z)<r /(2 A)$.

Secondly, if $d(y, z) \leq \sqrt{t}$, then using (1.1) and (5.1),

$$
\begin{aligned}
\frac{d(y, z)}{\sqrt{t}} & \lesssim\left(\frac{\mu(B(y, d(y, z))}{\mu(B(y, \sqrt{t}))}\right)^{1 / q} \\
& \leq\left(\frac{\mu(B(y, d(y, z))}{\mu(B(x, \sqrt{t}))}\right)^{1 / q}\left(\frac{\mu(B(y, \sqrt{t}+d(x, y))}{\mu(B(y, \sqrt{t}))}\right)^{1 / q} \\
& \lesssim\left(\frac{\tilde{d}(y, z)}{r}\right)^{1 / q}\left(1+\frac{d(x, y)}{\sqrt{t}}\right) .
\end{aligned}
$$

Thirdly, if $d(y, z) \geq \sqrt{t}$, then using (1.1) and (5.1),

$$
\begin{aligned}
1 & \lesssim \frac{\tilde{d}(y, z)}{\mu(B(y, \sqrt{t}))}=\frac{\tilde{d}(y, z)}{r} \frac{\mu(B(x, \sqrt{t})))}{\mu(B(y, \sqrt{t}))} \\
& \lesssim \frac{\widetilde{d}(y, z)}{r} \frac{\mu(B(y, \sqrt{t}+d(x, y)))}{\mu(B(y, \sqrt{t}))} \\
& \lesssim \frac{\widetilde{d}(y, z)}{r}\left(1+\frac{d(x, y)}{\sqrt{t}}\right)^{q} .
\end{aligned}
$$

Let us turn to the proof of (6.7).

Case 1: $d(y, z) \leq d(x, y) / 2$. Then $d(x, y) \simeq d(x, z)$. Thus, according to (1.3) and (4.3) combined with (6.8) and (6.9) we obtain

$$
\begin{aligned}
|\widetilde{T}(r, x, y)-\widetilde{T}(r, x, z)| & \lesssim r^{-1} \exp \left(-\frac{c d(x, y)^{2}}{t}\right) \min \left(\frac{d(y, z)}{\sqrt{t}}, 1\right)^{\alpha} \\
& \lesssim r^{-1} \exp \left(-\frac{c d(x, y)^{2}}{t}\right)\left(\frac{\tilde{d}(y, z)}{r}\right)^{\alpha / q}\left(1+\frac{d(x, y)}{\sqrt{t}}\right)^{\alpha} \\
& \lesssim r^{-1}\left(1+\frac{\tilde{d}(x, y)}{r}\right)^{-1-\delta}\left(\frac{\tilde{d}(y, z)}{r}\right)^{\alpha / q},
\end{aligned}
$$

where in the last inequality we have used (6.6).

Case 2: $d(y, z)>d(x, y) / 2$ and $\sqrt{t}>d(y, z)$. Then $\widetilde{d}(x, y) \lesssim \mu(B(x, \sqrt{t}))=r$. Using (4.3) and (6.8),

$$
|\widetilde{T}(r, x, y)-\widetilde{T}(r, x, z)| \lesssim r^{-1}\left(\frac{d(y, z)}{\sqrt{t}}\right)^{\alpha} \lesssim r^{-1}\left(\frac{\tilde{d}(y, z)}{r}\right)^{\alpha / q}
$$

Case 3: $d(y, z)>d(x, y) / 2$ and $\sqrt{t}<d(y, z)$. Then, $\tilde{d}(y, z) \leq r /(2 A) \leq r /\left(2 A_{2}\right)$ and by Lemma 17 we have $2 d(x, z)>d(x, y) \geq \sqrt{t}$. Hence, using (6.5) and (6.9),

$$
\begin{aligned}
|\widetilde{T}(r, x, y)-\widetilde{T}(r, x, z)| & \lesssim r^{-1} \exp \left(-\frac{c d(x, y)^{2}}{t}\right) \\
& \lesssim r^{-1} \exp \left(-\frac{c d(x, y)^{2}}{t}\right)\left(\frac{\widetilde{d}(y, z)}{r}\right)^{\alpha / q}\left(1+\frac{d(x, y)}{\sqrt{t}}\right)^{\alpha} \\
& \lesssim r^{-1}\left(\frac{\widetilde{d}(y, z)}{r}\right)^{\alpha / q}\left(1+\frac{\widetilde{d}(x, y)}{r}\right)^{-1-\delta},
\end{aligned}
$$

where in the last inequality we have used (6.6). This finishes the proof of Proposition 20. 
Proof of Theorem 2 Assuming (1.3) and (1.6) we obtain Hölder-type estimate (4.3). Recall once more that the assumption on $\Phi_{x}$ implies $\mu(X)=\infty$ and $\mu$ is nonatomic. Then we define a new quasi-metric $\widetilde{d}$. By Corollary 16 we get that $H_{\text {at }}^{1}(d, \mu)=H_{\text {at }}^{1}(\widetilde{d}, \mu)$. We apply Theorem 18 to the space $(X, \widetilde{d}, \mu)$. The assumptions of Theorem 18 are verified in Theorem 19 and Proposition 20. In this way we get

$$
\|f\|_{H^{1}(X, \widetilde{T})} \simeq\|f\|_{H_{\mathrm{at}}^{1}(\widetilde{d}, \mu)} .
$$

Using once again the assumption on $\Phi_{x}$ and the definition of $\widetilde{T}$ we easily observe that

$$
\|f\|_{H^{1}(X, \widetilde{T})}=\|f\|_{H^{1}(L)},
$$

which finishes the proof of Theorem 2.

Let us recall that Theorem 1 follows from Theorem 2. This is elaborated at the end of Sect. 3.

Remark 21 Under the assumptions of Theorem 1 one can prove, by the same methods, that the Hardy space

$$
H^{1}(\sqrt{L})=\left\{f \in L^{1}(X, \mu)\left|\|f\|_{H^{1}(\sqrt{L})}:=\left\|\sup _{t>0}\left|P_{t} f(x)\right|\right\|_{L^{1}(X, \mu)}<\infty\right\}\right.
$$

coincides with $H_{\mathrm{at}}^{1}(d, \omega \mu)$ and the corresponding norms are equivalent. To this end, one uses: Proposition 6, Doob's transform, Theorem 4, and Theorem 18 applied to the kernel $\widetilde{P}(r, x, y)=P_{t}(x, y)$, where $t=t(x, r)$ is defined by the relation $\mu(B(x, t))=r$.

\section{Examples}

In this section we give examples of self-adjoint semigroups with the two-sided Gaussian bounds.

\subsection{Laplace-Beltrami operators}

Let $(X, g)$ be a complete Riemannian manifold with the Riemannian distance $d(x, y)$ and the Riemannian measure $\mu$ satisfying the doubling property and the Poincare inequality

$$
\int_{B(x, r)}\left|f-f_{B}\right|^{2} \mathrm{~d} \mu \leq C r^{2} \int_{B(x, 2 r)}|\nabla f|^{2} \mathrm{~d} \mu \quad \text { for all } r>0,
$$

where $\nabla$ denotes the gradient on $X$. It is well known that the kernel of the heat semigroup generated by the Laplace-Beltrami operator satisfies two-sided Gaussian bounds (1.3) and Hölder estimates (4.3). For details and more information concerning the heat equation on Riemannian manifolds we refer the reader to [28] and references therein.

\subsection{Schrödinger operators}

On $X=\mathbb{R}^{d}$ with the Euclidean metric and the Lebesgue measure we consider the Schrödinger operator

$$
L=-\Delta+V,
$$

where $\Delta$ is the standard Laplacian and $V$ is a locally integrable function. 
It is well known (see, e.g., [29]) that for $V \geq 0, d \geq 3$, the semigroup $T_{t}=e^{-t L}$ admits kernels $T_{t}(x, y)$ with the upper and lower Gaussian bounds (1.3) if and only if $V$ is a Green bounded potential, that is,

$$
\sup _{x \in \mathbb{R}^{d}} \int_{\mathbb{R}^{d}}|x-y|^{2-d} V(y) d y<\infty .
$$

Hardy spaces associated with Schrödinger operators on $\mathbb{R}^{d}$ satisfying (7.1) were studied in [13]. Actually, as we have already mentioned, this work motivated us to study the problem of $H^{1}$ spaces with the Gaussian bounds in the generality as in Theorems 1 and 2.

Our second example concerns Schrödinger operators $L=-\Delta+V$ with nonpositive potentials. For $d \geq 3$ fix $p_{1}, p_{2}>1$ satisfying $p_{1}<d / 2<p_{2}$. Then there is a constant $c\left(p_{1}, p_{2}, d\right)>0$ such that if $V \leq 0$ and

$$
\|V\|_{L^{p_{1}\left(\mathbb{R}^{d}\right)}}+\|V\|_{L^{p_{2}\left(\mathbb{R}^{d}\right)}} \leq c\left(p_{1}, p_{2}, d\right),
$$

then the integral kernel $T_{t}(x, y)$ of the semigroup $T_{t}=e^{-t L}$ exists and satisfies two-sided Gaussian bounds

$$
T_{t}(x, y) \simeq t^{-d / 2} \exp \left(-\frac{|x-y|^{2}}{4 t}\right) .
$$

The result can be found in Zhang [35]. A slightly different proof of (7.2), based on bridges of the Gauss-Weierstrass semigroup, can be obtained by using Lemma 1.2 together with Proposition 2.2 of [4].

\subsection{Bessel-Schrödinger operator}

Let $\alpha>-1$ and consider $X=(0, \infty)$ and $\mathrm{d} \mu(x)=x^{\alpha} d x$. Notice that the space $(X, \mu)$ with the Euclidean metric $d_{e}(x, y)=|x-y|$ is a space of homogeneous type. We consider the classical Bessel operator

$$
\mathcal{B} f(x)=-f^{\prime \prime}(x)-\frac{\alpha}{x} f^{\prime}(x)
$$

which is self-adjoint positive on $L^{2}(X, \mu)$, and the associated semigroup of linear operators $S_{t}=e^{-t \mathcal{B}}$. It is well known that $S_{t}$ is conservative and has the integral kernel

$$
S_{t}(x, y)=(2 t)^{-1} \exp \left(-\frac{x^{2}+y^{2}}{4 t}\right) I_{(\alpha-1) / 2}\left(\frac{x y}{2 t}\right)(x y)^{-(\alpha-1) / 2},
$$

see, e.g., [5, Chapter 6]. Here $I_{(\alpha-1) / 2}$ denotes the modified Bessel function of the first kind, see, e.g., [34]. The kernel $S_{t}(x, y)$ satisfies two-sided Gaussian estimates

$$
\frac{C_{0}^{-1}}{\mu(B(x, \sqrt{t}))} \exp \left(-\frac{c_{1}|x-y|^{2}}{t}\right) \leq S_{t}(x, y) \leq \frac{C_{0}}{\mu(B(x, \sqrt{t}))} \exp \left(-\frac{c_{2}|x-y|^{2}}{t}\right)
$$

For a short proof of (7.4) see [12, proof of Lemma 4.2]. Therefore, using Theorem 2 we obtain atomic characterization of $H^{1}(\mathcal{B})$ that was previously proved in [3].

In this subsection we consider perturbations of $\mathcal{B}$ of the form

$$
L=\mathcal{B}+V
$$


where a potential $V$ is nonnegative and locally integrable. More precisely, on $L^{2}(X, \mu)$ we define the quadratic form

$$
Q(f, g)=\int_{X} f^{\prime}(x) \overline{g^{\prime}(x)} \mathrm{d} \mu(x)+\int_{X} V(x) f(x) \overline{g(x)} \mathrm{d} \mu(x),
$$

with the domain

$$
\operatorname{Dom}(Q)=\operatorname{cl}\left\{f \in C_{c}^{1}[0, \infty) \mid f^{\prime}\left(0^{+}\right)=0\right\} \cap\left\{f \in L^{2}(X, \mu) \mid \sqrt{V} f \in L^{2}(X, \mu)\right\},
$$

where $\operatorname{cl}(A)$ stands for the closure of the set $A$ in the norm $\|f\|_{L^{2}(X, \mu)}+\left\|f^{\prime}\right\|_{L^{2}(X, \mu)}$. The form $Q$ is positive and closed. Thus, it corresponds to the unique self-adjoint operator $L$ on $L^{2}(X, \mu)$ with the domain

$\operatorname{Dom}(L)=\left\{f \in \operatorname{Dom}(Q) \mid\left(\exists h \in L^{2}(X, \mu)\right)(\forall g \in \operatorname{Dom}(Q)) Q(f, g)=\int h \bar{g} \mathrm{~d} \mu\right\}$.

By definition, $L f=h$ when $f, h$ are related as above.

Let $T_{t}=\exp (-t L)$ be the semigroup generated by $-L$. The Feynman-Kac formula states that

$$
T_{t} f(x)=E^{x}\left(\exp \left(-\int_{0}^{t} V\left(b_{s}\right) \mathrm{d} s\right) f\left(b_{t}\right)\right),
$$

where $b_{s}$ is the Bessel process on $(0, \infty)$ associated with $S_{t}$. Using (7.5) one gets that the semigroup $T_{t}$ has form (1.2), where

$$
0 \leq T_{t}(x, y) \leq S_{t}(x, y) .
$$

Therefore the upper Gaussian estimates for $T_{t}(x, y)$ follows simply from (7.4) for any locally integrable $V \geq 0$. On the other hand, the relation between $S_{t}(x, y)$ and $T_{t}(x, y)$ is given by the perturbation formula

$$
S_{t}(x, y)=T_{t}(x, y)+\int_{0}^{t} \int_{X} S_{t-s}(x, z) V(z) T_{s}(z, y) \mathrm{d} \mu(z) \mathrm{d} s
$$

From now on we consider only $\alpha>1$. We are interested in proving the lower Gaussian estimates, but this can be done only for some potentials $V$. For other potentials Hardy spaces may have a local character, (see, e.g., [22]). Let

$$
\Gamma(x, y)=\int_{0}^{\infty} S_{t}(x, y) \mathrm{d} t
$$

be the formal kernel of $\mathcal{B}^{-1}$. In addition to $V \in L_{l o c}^{1}(X)$ and $V \geq 0$, we shall need one more assumption, a version of the global Kato condition, cf. (7.1),

$$
\|V\|_{\text {Kato }}:=\sup _{x \in X} \int_{X} \Gamma(x, y) V(y) \mathrm{d} \mu(y)<\infty .
$$

Formally, we can rephrase this as $\mathcal{B}^{-1} V \in L^{\infty}(X, \mu)$. Let us point out that

$$
\Gamma(x, y) \simeq(x+y)^{-\alpha+1} .
$$

This can be easily obtained from (7.3) and well-known asymptotics for the Bessel function $I_{(\alpha-1) / 2}$, see also [26, Sect. 2].

In Lemmas 22 and 23 we prove that under assumption (7.8) lower Gaussian estimates (1.3) hold for $T_{t}(x, y)$. The estimates are proved in a similar way as in the case of the Schrödinger operator on the Euclidean space. For the convenience of the reader we provide the details. 
Lemma 22 Suppose that $\|V\|_{\text {Kato }}<\infty$. If $|x-y| \leq \sqrt{t}$, then

$$
T_{t}(x, y) \geq C_{l}^{-1} \mu(B(x, \sqrt{t}))^{-1} .
$$

Proof First we shall prove Lemma 22 with an additional assumption that $\|V\|_{\text {Kato }} \leq \varepsilon$ for a fixed small $\varepsilon>0$. By (7.7) and (7.6),

$$
\begin{aligned}
S_{t}(x, y)-T_{t}(x, y)= & \int_{0}^{t} \int_{X} S_{t-s}(x, z) V(z) T_{s}(z, y) \mathrm{d} \mu(z) \mathrm{d} s=\int_{0}^{t / 2} \ldots \mathrm{d} s+\int_{t / 2}^{t} \ldots \mathrm{d} s \\
\lesssim & \mu(B(x, \sqrt{t}))^{-1} \int_{0}^{t / 2} \int_{X} V(z) T_{s}(z, y) \mathrm{d} \mu(z) \mathrm{d} s \\
& +\mu(B(y, \sqrt{t}))^{-1} \int_{t / 2}^{t} \int_{X} S_{t-s}(x, z) V(z) \mathrm{d} \mu(z) \mathrm{d} s \\
\lesssim & \mu(B(x, \sqrt{t}))^{-1}\|V\|_{\text {Kato }} .
\end{aligned}
$$

By choosing proper $\varepsilon>0$ we deduce the thesis from lower estimate (7.4) for $S_{t}(x, y)$.

Now assume that the norm $\|V\|_{\text {Kato }}<\infty$ is arbitrary. Fix $q>1$, such that $\|V\|_{\text {Kato }}=q \varepsilon$. Set $V_{q}(x)=V(x) / q,\left\|V_{q}\right\|_{\text {Kato }}=\varepsilon$, and let $T_{t}^{q}$ be the semigroup related to $L_{q}=\mathcal{B}+V_{q}$. Using (7.5) and Hölder's inequality,

$$
\begin{aligned}
T_{t}\left(\frac{\chi_{B(y, r)}(\cdot)}{\mu(B(y, r))}\right)(x) & =E^{x}\left(\left(\exp \left(-\int_{0}^{t} \frac{V\left(b_{s}\right)}{q} \mathrm{~d} s\right)\right)^{q} \frac{\chi_{B(y, r)}\left(b_{t}\right)}{\mu(B(y, r))}\right) \\
& \geq\left[E^{x}\left(\exp \left(-\int_{0}^{t} \frac{V\left(b_{s}\right)}{q} \mathrm{~d} s\right) \frac{\chi_{B(y, r)}\left(b_{t}\right)}{\mu(B(y, r))}\right)\right]^{q}\left[E^{x}\left(\frac{\chi_{B(y, r)}\left(b_{t}\right)}{\mu(B(y, r))}\right)\right]^{-\frac{q}{q^{\prime}}} \\
& =\left[T_{t}^{q}\left(\frac{\chi_{B(y, r)}(\cdot)}{\mu(B(y, r))}\right)(x)\right]^{q} \cdot\left[S_{t}\left(\frac{\chi_{B(y, r)}(\cdot)}{\mu(B(y, r))}\right)(x)\right]^{-q / q^{\prime}}
\end{aligned}
$$

Let us notice that

$$
T_{t}(x, y)=\lim _{r \rightarrow 0} \mu(B(y, r))^{-1} \int_{B(y, r)} T_{t}(x, z) \mathrm{d} \mu(z)
$$

for a.e. $(x, y)$. By letting $r \rightarrow 0$ in (7.9), using (7.4) and the first part of the proof, for a.e. $(x, y)$ we obtain that

$$
T_{t}(x, y) \geq T_{t}^{q}(x, y)^{q} S_{t}(x, y)^{-q / q^{\prime}} \gtrsim \mu(B(x, \sqrt{t}))^{-1} .
$$

Lemma 23 Suppose that $\|V\|_{\text {Kato }}<\infty$. Then

$$
T_{t}(x, y) \gtrsim \mu(B(x, \sqrt{t}))^{-1} \exp \left(-\frac{c|x-y|^{2}}{t}\right) .
$$

Proof Assume that $|x-y|^{2} / t \geq 1$ and set $m=\left\lceil 4|x-y|^{2} / t\right\rceil \geq 4$. For $i=0, \ldots, m$, let $x_{i}=x+i(y-x) / m$, so that $x_{0}=x, x_{m}=y$, and $\left|x_{i+1}-x_{i}\right|=|x-y| / m$. Denote $B_{i}=B\left(x_{i}, \sqrt{t} /(4 \sqrt{m})\right)$ and observe that

$$
\left|y_{i+1}-y_{i}\right| \leq\left|y_{i}-x_{i}\right|+\left|x_{i}-x_{i+1}\right|+\left|x_{i+1}-y_{i+1}\right| \leq \frac{\sqrt{t}}{4 \sqrt{m}}+\frac{\sqrt{t}}{2 \sqrt{m}}+\frac{\sqrt{t}}{4 \sqrt{m}}=\frac{\sqrt{t}}{\sqrt{m}}
$$


for $y_{i} \in B_{i}$ and $y_{i+1} \in B_{i+1}$. Now we use the semigroup property, Lemma 22, and the doubling property of $\mu$ to obtain

$$
\begin{aligned}
& T_{t}(x, y)=\int_{X} \ldots \int_{X} T_{t / m}\left(x, y_{1}\right) T_{t / m}\left(y_{1}, y_{2}\right) \ldots T_{t / m}\left(y_{m-1}, y\right) \mathrm{d} \mu\left(y_{1}\right) \ldots \mathrm{d} \mu\left(y_{m-1}\right) \\
& \geq c_{1}^{m-1} \int_{B_{1}} \ldots \int_{B_{m-1}} \mu(B(x, \sqrt{t / m}))^{-1} \ldots \mu\left(B\left(y_{m-1}, \sqrt{t / m}\right)\right)^{-1} \mathrm{~d} \mu\left(y_{1}\right) \ldots \mathrm{d} \mu\left(y_{m-1}\right) \\
& \geq c_{2}^{m-1} \mu(B(x, \sqrt{t / m}))^{-1} \frac{\mu\left(B_{1}\right) \ldots \mu\left(B_{m-1}\right)}{\mu\left(B\left(x_{1}, \sqrt{t / m}\right)\right) \ldots \mu\left(B\left(x_{m-1}, \sqrt{t / m}\right)\right)} \\
& \geq c_{3}^{m} \mu(B(x, \sqrt{t}))^{-1}=\mu(B(x, \sqrt{t}))^{-1} e^{-m \ln c_{3}^{-1}} \gtrsim \mu(B(x, \sqrt{t}))^{-1} e^{-\frac{c|x-y|^{2}}{t}} .
\end{aligned}
$$

Notice that $c_{1}, c_{2}, c_{3}$, and $c$ in this estimate depend only on the constant $C_{l}$ from Lemma 22 and the doubling constant of $\mu$.

Obviously, the space $\left(X, d_{e}, \mu\right)$ satisfies the assumptions of Theorem 1 . Since we have the two-sided Gaussian estimates for $T_{t}$ (see Lemma 22, (7.6), and (7.4)) we obtain the following corollary.

Corollary 24 Suppose that $\alpha>1$ and $V \geq 0$ satisfies (7.8). Then there exists $\omega$ such that $0<C^{-1} \leq \omega(x) \leq C, T_{t} \omega=\omega$ for $t>0$ and $H^{1}(L)=H_{\mathrm{at}}^{1}\left(d_{e}, \omega \mu\right)$. Moreover,

$$
\|f\|_{H^{1}(L)} \simeq\|f\|_{H_{\mathrm{at}}^{1}\left(d_{e}, \omega \mu\right)} .
$$

Acknowledgements We want to thank Pascal Aucher, Frédéric Bernicot, Li Chen, and Grzegorz Plebanek for their remarks. We are greatly indebt to Jacek Zienkiewicz for conversations concerning Schrödinger operators. The authors are grateful to the referee for her/his helpful comments which improved the presentation the paper.

Open Access This article is distributed under the terms of the Creative Commons Attribution 4.0 International License (http://creativecommons.org/licenses/by/4.0/), which permits unrestricted use, distribution, and reproduction in any medium, provided you give appropriate credit to the original author(s) and the source, provide a link to the Creative Commons license, and indicate if changes were made.

\section{References}

1. Auscher, P., Duong, X.T., McIntosh, A.: Boundedness of banach space valued singular integral operators and hardy spaces. Unpublished preprint (2005)

2. Bernicot, F., Coulhon, T., Frey, D.: Gaussian heat kernel bounds through elliptic Moser iteration. J. Math. Pures Appl. (9) 106(6), 995-1037 (2016)

3. Betancor, J.J., Dziubański, J., Torrea, J.L.: On Hardy spaces associated with Bessel operators. J. Anal. Math. 107, 195-219 (2009)

4. Bogdan, K., Dziubański, J., Szczypkowski, K.: Sharp Gaussian estimates for Schrödinger heat kernels: $L^{p}$ integrability conditions. ArXiv e-prints (2015)

5. Borodin, A.N., Salminen, P.: Handbook of Brownian Motion-Facts and Formulae. Probability and its Applications, 2nd edn. Birkhäuser, Basel (2002)

6. Burkholder, D.L., Gundy, R.F., Silverstein, M.L.: A maximal function characterization of the class $H^{p}$. Trans. Am. Math. Soc. 157, 137-153 (1971)

7. Coifman, R.R.: A real variable characterization of $H^{p}$. Stud. Math. 51, 269-274 (1974)

8. Coifman, R.R., Weiss, G.: Extensions of Hardy spaces and their use in analysis. Bull. Am. Math. Soc. 83(4), 569-645 (1977)

9. Coulhon, T., Sikora, A.: Gaussian heat kernel upper bounds via the Phragmén-Lindelöf theorem. Proc. Lond. Math. Soc. (3) 96(2), 507-544 (2008)

10. Davies, E.B.: Heat Kernels and Spectral Theory, Cambridge Tracts in Mathematics, vol. 92. Cambridge University Press, Cambridge (1990)

11. Dziubański, J., Preisner, M.: Remarks on spectral multiplier theorems on Hardy spaces associated with semigroups of operators. Rev. Un. Mat. Argentina 50(2), 201-215 (2009) 
12. Dziubański, J., Preisner, M., Wróbel, B.: Multivariate Hörmander-type multiplier theorem for the Hankel transform. J. Fourier Anal. Appl. 19(2), 417-437 (2013)

13. Dziubański, J., Zienkiewicz, J.: On isomorphisms of Hardy spaces associated with Schrödinger operators. J. Fourier Anal. Appl. 19(3), 447-456 (2013)

14. Dziubański, J., Zienkiewicz, J.: A characterization of Hardy spaces associated with certain Schrödinger operators. Potential Anal. 41(3), 917-930 (2014)

15. Fefferman, C., Stein, E.M.: $H^{p}$ spaces of several variables. Acta Math. 129(3-4), 137-193 (1972)

16. Grigor'yan, A.: Heat Kernel and Analysis on Manifolds, AMS/IP Studies in Advanced Mathematics, vol. 47. American Mathematical Society, International Press, Providence, Boston (2009)

17. Gyrya, P., Saloff-Coste, L.: Neumann and Dirichlet heat kernels in inner uniform domains. Astérisque 336, viii+144 (2011)

18. Hebisch, W.: Functional calculus for slowly decaying kernels. Unpublished. http://www.math.uni.wroc. $\mathrm{pl} / \sim$ hebisch/

19. Hebisch, W., Saloff-Coste, L.: On the relation between elliptic and parabolic Harnack inequalities. Ann. Inst. Fourier (Grenoble) 51(5), 1437-1481 (2001)

20. Hofmann, S., Lu, G., Mitrea, D., Mitrea, M., Yan, L.: Hardy spaces associated to non-negative self-adjoint operators satisfying Davies-Gaffney estimates. Mem. Am. Math. Soc. 214(1007), vi+78 (2011)

21. Jerison, D.S., Kenig, C.E.: Boundary value problems on Lipschitz domains, Studies in partial differential equations, MAA Stud. Math., vol. 23. Mathematical Association of America, Washington pp. 1-68 (1982)

22. Kania, E., Preisner, M.: Hardy spaces for Bessel-Schrödinger operators. arXiv e-prints (2016)

23. Latter, R.H.: A characterization of $H^{p}\left(\mathbf{R}^{n}\right)$ in terms of atoms. Stud. Math. 62(1), 93-101 (1978)

24. Macías, R.A., Segovia, C.: A decomposition into atoms of distributions on spaces of homogeneous type. Adv. Math. 33(3), 271-309 (1979)

25. Macías, R.A., Segovia, C.: Lipschitz functions on spaces of homogeneous type. Adv. Math. 33(3), 257270 (1979)

26. Nowak, A., Stempak, K.: Potential operators associated with Hankel and Hankel-Dunkl transforms. J. Anal. Math. 131, 277-321 (2017)

27. Ouhabaz, E.M.: Analysis of Heat Equations on Domains, London Mathematical Society Monographs Series, vol. 31. Princeton University Press, Princeton (2005)

28. Saloff-Coste, L.: Aspects of Sobolev-Type Inequalities, London Mathematical Society Lecture Note Series, vol. 289. Cambridge University Press, Cambridge (2002)

29. Semenov, YuA: Stability of $L^{p}$-spectrum of generalized Schrödinger operators and equivalence of Green's functions. Int. Math. Res. Not. 12, 573-593 (1997)

30. Song, L., Yan, L.: A maximal function characterization for Hardy spaces associated to nonnegative selfadjoint operators satisfying Gaussian estimates. Adv. Math. 287, 463-484 (2016). https://doi.org/10. 1016/j.aim.2015.09.026

31. Stein, E.M.: Harmonic Analysis: Real-Variable Methods, Orthogonality, and Oscillatory Integrals. Princeton Mathematical Series, vol. 43. Princeton University Press, Princeton (1993). (With the assistance of Timothy S. Murphy, Monographs in Harmonic Analysis, III)

32. Stein, E.M., Weiss, G.: On the theory of harmonic functions of several variables. I. The theory of $H^{p}$ spaces. Acta Math. 103, 25-62 (1960)

33. Uchiyama, A.: A maximal function characterization of $H^{p}$ on the space of homogeneous type. Trans. Am. Math. Soc. 262(2), 579-592 (1980)

34. Watson, G.N.: A Treatise on the Theory of Bessel Functions, Cambridge Mathematical Library. Cambridge University Press, Cambridge (1995). (Reprint of the second (1944) edition)

35. Zhang, Q.S.: A sharp comparison result concerning Schrödinger heat kernels. Bull. Lond. Math. Soc. 35(4), 461-472 (2003) 